\title{
Non-parsimonious evolution of hagfish Dlx genes
}

\author{
Satoko Fujimoto ${ }^{1}$, Yasuhiro Oisi ${ }^{1,2}$, Shigehiro Kuraku ${ }^{3}$, Kinya G Ota ${ }^{4^{*}}$ and Shigeru Kuratani ${ }^{1}$
}

\begin{abstract}
Background: The number of members of the Dlx gene family increased during the two rounds of whole-genome duplication that occurred in the common ancestor of the vertebrates. Because the D/x genes are involved in the development of the cranial skeleton, brain, and sensory organs, their expression patterns have been analysed in various organisms in the context of evolutionary developmental biology. Six D/x genes have been isolated in the lampreys, a group of living jawless vertebrates (cyclostomes), and their expression patterns analysed. However, little is known about the D/x genes in the hagfish, the other cyclostome group, mainly because the embryological analysis of this animal is difficult.

Results: To identify the hagfish Dlx genes and describe their expression patterns, we cloned the cDNA from embryos of the Japanese inshore hagfish Eptatretus burgeri. Our results show that the hagfish has at least six D/x genes and one pseudogene. In a phylogenetic analysis, the hagfish D/x genes and those of the lampreys tended to be excluded from the clade of the gnathostome D/x genes. In several cases, the lamprey D/x genes clustered with the clade consisting of two hagfish genes, suggesting that independent gene duplications have occurred in the hagfish lineage. Analysis of the expression of these genes showed distinctive overlapping expression patterns in the cranial mesenchymal cells and the inner ear.

Conclusions: Independent duplication, pseudogenization, and loss of the D/x genes probably occurred in the hagfish lineage after its split from the other vertebrate lineages. This pattern is reminiscent of the non-parsimonious evolution of its morphological traits, including its inner ear and vertebrae, which indicate that this group is an early-branching lineage that diverged before those characters evolved.
\end{abstract}

Keywords: Cyclostomes, Hagfish embryos, D/x genes, Gene duplication

\section{Background}

The extant vertebrates are divided into two major groups, the jawed (gnathostomes) and the jawless vertebrates (agnathans). The two groups share a number of morphological characters (synapomorphies) that define the vertebrates, such as the neurogenic placode, neural crest, and their derivatives, including complex sense organs and a cranial skeleton [1-3]. These morphological characters are not seen in non-vertebrate chordates. To investigate the early phase of vertebrate evolution from a molecular perspective, the expression patterns of various developmental regulatory genes have been compared between the gnathostomes and the lamprey, one of the two extant groups of agnathans [4-14]. In contrast to the lamprey, little is known about the developmental

\footnotetext{
* Correspondence: otakinya@gate.sinica.edu.tw

${ }^{4}$ Laboratory of Aquatic Zoology, Marine Research Station, Institute of Cellular and Organismic Biology, Academia Sinica, Yilan 26242, Taiwan

Full list of author information is available at the end of the article
}

processes of these morphological characters in the hagfish because their embryos have been unavailable until recently.

The cyclostomes are often recognized as a paraphyletic group in the fields of morphology and palaeontology $[3,15-17]$ because of the extraordinarily different morphologies of the hagfish and lampreys $[18,19]$. In fact, the hagfish has been considered to lack a number of the vertebrate characters possessed by the lamprey, such as deepithelialized and migrating neural crest cells, vertebral elements, a complex branchial basket, and multiple semicircular canals in the inner ear [18-22]. Based on the idea that these relatively simple morphological features of the hagfish represent the ancestral state of the vertebrates, this animal has tended to be placed at the base of the phylogenetic tree of the entire vertebrates [3,15-17]. However, on various molecular phylogenetic trees, the hagfish tends to cluster with the lamprey in a monophyletic group, and this position is now widely

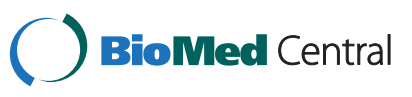


accepted by researchers who are familiar with these molecular phylogenetic analyses [23-27]. This discrepancy between the molecular and morphological data has been a source of contention regarding the evolution of the early vertebrates, and there was no consensus on the phylogenetic position of the hagfish for about three decades [28].

However, in this century, the situation in the field of hagfish research has changed. Since 2007, a number of live embryos of the Japanese inshore hagfish, Eptatretus burgeri, have been obtained (Figure 1), and these have provided an opportunity to conduct intensive molecular cloning and analysis of the hagfish embryonic gene expression patterns [29-31]. Even more significantly, these molecular studies of hagfish embryos have demonstrated that, contrary to the suggestions of traditional text-

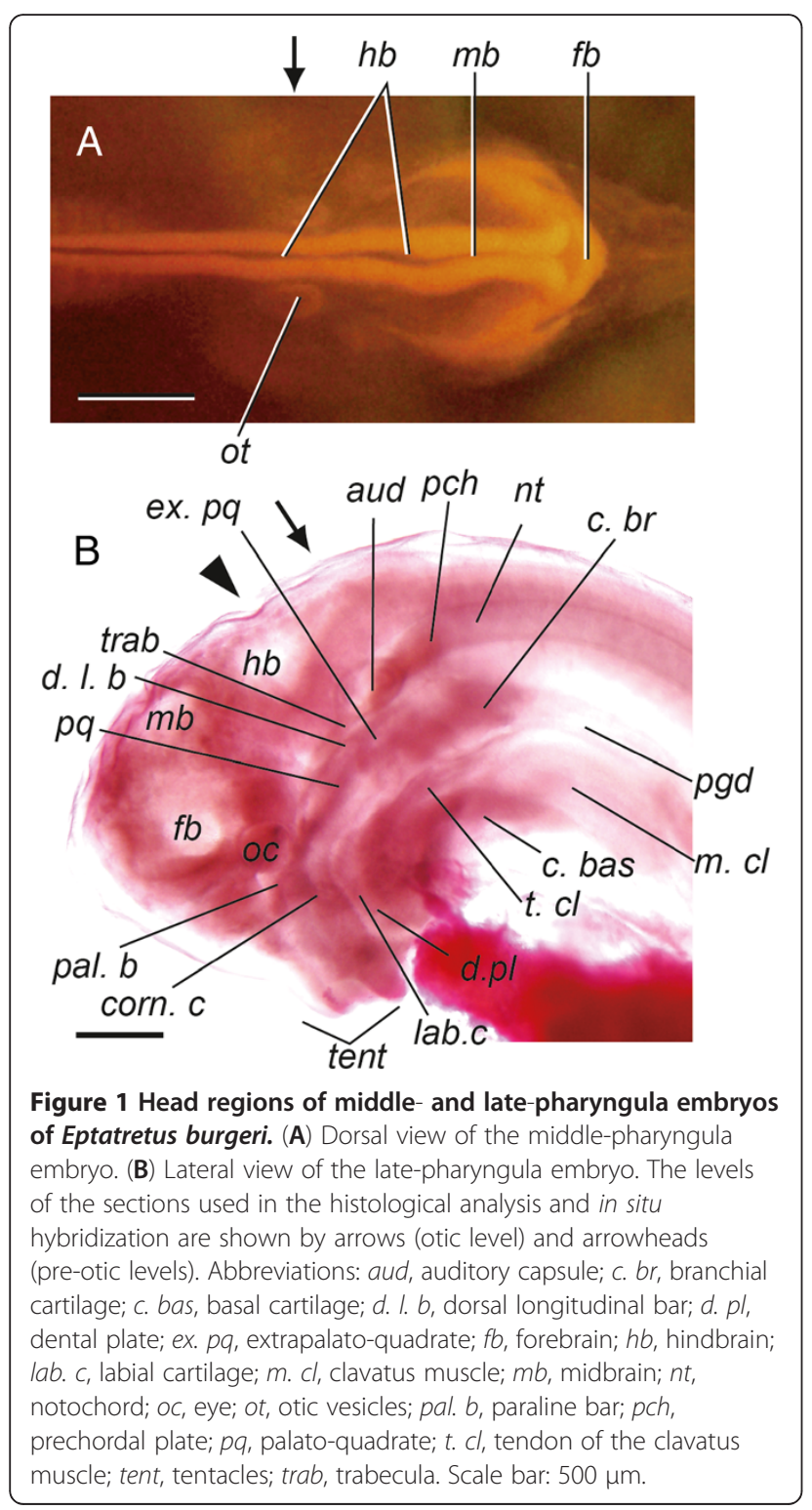

books, the hagfish has de-epithelialized and migrating neural crest cells and vertebral elements [19,30-33]. Furthermore, a comparative analysis of the hagfish and lamprey microRNAs tended to support a monophyletic relationship between them [34]. These lines of evidence seem to have established a consensus among molecular biologists and palaeontologists on the phylogenetic position of the hagfish. In fact, in a recently published paleontological report on the evolution of the early vertebrates, the hagfish is recognized as the sister group of the lampreys [35]. Therefore, it is now possible to reconstruct a more plausible evolutionary scenario of how the morphology of the hagfish diverged from that of the other vertebrates, by examining the embryonic events of this animal with molecular biological techniques.

Given the monophyly of the cyclostomes, it is conceivable that the molecular developmental mechanisms of the lampreys and hagfish evolved independently in each lineage after their divergence more than 400 million years ago [36], resulting in secondarily degenerate characters that are more marked in the hagfish lineage. In fact, this assumption is consistent, at the molecular level, with the evidence that the Xlox gene, one of the ParaHox genes responsible for organogenesis (including pancreas formation) in the gnathostomes, is pseudogenized in the genome of the Atlantic hagfish (Myxine glutinosa), which may correlate with the absence of some endocrine organs in the hagfish [37]. This suggests that some molecular sequences and expression patterns could be secondarily degenerate for other hagfish genes that are involved in the developmental processes of the vertebrate morphological synapomorphies.

Because the $D l x$ genes are crucial to the morphogenesis of the vertebrate synapomorphies, they may also be secondarily degenerate in the hagfish [38-42]. The $D l x$ genes, homeobox-containing transcription factors, are organized in convergently transcribed bi-gene clusters, which are linked to the Hox gene clusters in the genomes of the gnathostomes. For example, the six $D l x$ genes of mammals form three bi-gene clusters, $D l x 1-$ $D l \times 2, D l x 3-D l x 4$, and $D l x 5-D l x 6$, linked to the HoxD, $H o x B$, and HoxA clusters, respectively [43-47]. From the evidence that the chondrichthyan species have three $D l x$ bi-gene clusters, it is presumed that the common ancestor of the gnathostomes already had three bi-gene clusters, which seem to have derived from two rounds of genome duplication [44,48]. More significantly, the $D l x$ genes show overlapping expression patterns in the synapomorphic characters of the gnathostomes; for example, in the forebrain, neural crest cells, and inner ear [40-42]. Almost all the $D l x$ genes are expressed in the ectomesenchymal cells derived from the cranial neural crest $[41,49,50]$, and the expression of some $D l x$ genes is detected during the developmental processes of the 
inner ear $[40,42]$ in model gnathostome species, including the chicken, mouse, and zebrafish.

It has been demonstrated that the lamprey has at least six $D l x$ genes, designated $D l x A-F[13,51]$. At least four of these genes were generated by independent duplications unique to the lineage of either the lampreys or the cyclostomes. It is also known that $D l x A-E$ display overlapping expression patterns in the cranial ectomesenchyme and some of them in the otic vesicles $[7,13,14]$. These data raise several simple questions. How many $D l x$ genes are there in the hagfish genome? Did the hagfish ancestor also undergo an independent duplication of the $D l x$ genes, as in the lamprey lineage?

The derivatives of the cranial ectomesenchyme and the otic vesicle are also clearly simpler in the hagfish than in the lampreys and gnathostomes at the morphological level $[18,30]$. For example, the basket-like structure of the branchial skeleton is present in the lampreys but absent in the hagfish. There is also no vertical semicircular canal in the hagfish inner ear $[18,19]$. These lines of evidence raise another question. Do the $D l x$ genes of the hagfish also show overlapping expression patterns in the cranial ectomesenchyme and otic vesicles, similar to those in the lamprey and gnathostomes?

To address these questions, we cloned the $D l x$ genes from embryonic materials of the Japanese inshore hagfish (E. burgeri) and analysed the gene expression patterns of the isolated hagfish $D l x$ genes using in situ hybridization. Here, we show that the hagfish has at least six $D l x$ genes, some of which arose from gene duplications unique to the hagfish lineage. Furthermore, some of the isolated hagfish $D l x$ genes are expressed in the cranial mesenchymal cells and otic vesicles with overlapping expression patterns, as reported in the lampreys and gnathostomes $[6,7,13,41,42]$. These results suggest that the $D l x$ genes were independently duplicated and then diverged in each of the hagfish and lamprey lineages, maintaining overlapping expression patterns.

\section{Results}

\section{Identification of hagfish Dlx-encoding cDNAs}

To isolate full-length cDNAs containing the $D l x$ genes from the hagfish, we used degenerate RT-PCR and $5^{\prime} / 3^{\prime}$ RACE-PCR to amplify seven candidate genes from the embryonic material of E. burgeri (see Methods). All these isolated cDNAs contained nucleotide stretches corresponding to homeodomains, and one of them had a stop codon in the homeodomain (see Additional file 1). In our sequence comparison and preliminary molecular phylogenetic analysis, these genes were classified into two major groups (the $D l \times 1 / 4 / 6$ and $D l \times 2 / 3 / 5$ clades; Figure $2 \mathrm{~A}$ ). Because of the paucity of informative amino acid sites and the unstable positions of the outgroups, we could not identify the strict orthology of the isolated hagfish genes with the gnathostome $D l x$ genes. Therefore, we categorized and designated these seven genes from $E$. burgeri $E b D l x 1 / 4 / 6 A$, $E b D l x 1 / 4 / 6 B, E b D l x 1 / 4 / 6 C, E b D l \times 2 / 3 / 5 A, E b D l \times 2 / 3 / 5 B$, $E b D l x 2 / 3 / 5 C$, and $E b D l x \Psi$, according to the sequence similarities of the homeodomains and their flanking regions and the topology of the phylogenetic tree.

From a comparison of the conserved regions of the amino acid sequences, it is expected that $E b D l x \Psi$ and $E b D l \times 2 / 3 / 5 B$ are closely related to each other, implying that they diverged recently in the hagfish lineage. However, we could not identify the phylogenetic position of this pseudogene from our data set, because of the paucity of informative amino acid site between $E b D l x \Psi$ and the other sequences (see Additional file 1). Therefore, we excluded $E b D l x \Psi$ from our phylogenetic analysis.

\section{Molecular phylogeny of the hagfish Dlx genes}

Molecular phylogenetic trees of the vertebrate $D l x$ genes were reconstructed using their homologues in two tunicate species (Ciona intestinalis and Oikopleura dioica) as outgroups. Although the positions of the tunicate $D l x$ homologues were not stable, these genes were excluded from the clades of the gnathostome and cyclostome $D l x$ genes in our phylogenetic trees (Figure 2). These outgroups are positioned on the internal branch connecting the $D l x 1 / 4 / 6$ and $D l x 2 / 3 / 5$ gene clades of the vertebrates on a phylogenetic tree consisting of 68 operational taxonomic units (Figure 2A). On this phylogenetic tree, $E b D l x 1 / 4 / 6 C$ forms a cluster with $D l x F$ of the lamprey with strong support, $E b D l \times 2 / 3 / 5 A$ and $E b D l \times 2 / 3 / 5 B$ cluster in a clade containing three lamprey genes $(D l x A$, $-B$, and $-C$ ), and $E b D l \times 1 / 4 / 6 A$ and $-B$ cluster with two lamprey genes $(D l x D$ and $-E$ ) (Figure $2 A)$. Exceptionally, $E b D l \times 2 / 3 / 5 C$ was isolated from the other cyclostome $D l x$ genes on this phylogenetic tree.

To increase the number of informative amino acid sites for the phylogenetic analysis and to improve the resolution of the phylogenetic tree, we separately analysed the $D l \times 1 / 4 / 6$ and $D l \times 2 / 3 / 4$ clade genes with/without the outgroups (Figure 2B, C; Additional File 2). The clade of $E b D l x 1 / 4 / 6 C$ and the lamprey $D l x F$ gene was located at the most basal position of the vertebrate $D l x 1 / 4 / 6$ gene clade on the phylogenetic tree that included the outgroups (Figure 2B). To maximize the number of informative amino acid sites used in the phylogenetic inference, we excluded the sequences that produced extremely long branches (all tunicate $D l x$ homologues, gnathostome $D l x 4$ and $-6, E b D l x 1 / 4 / 6 C$, and lamprey $D l x F$ genes) from the alignment. On this phylogenetic tree, four $D l x$ genes of the cyclostomes formed a single clade, in which two $E b D l x 1 / 4 / 6$ genes clustered with a high bootstrap value (Additional file 2A). The cluster containing the lamprey $D l x D$ and $D l x E$ genes was located next to the hagfish cluster, although the bootstrap value was not very 

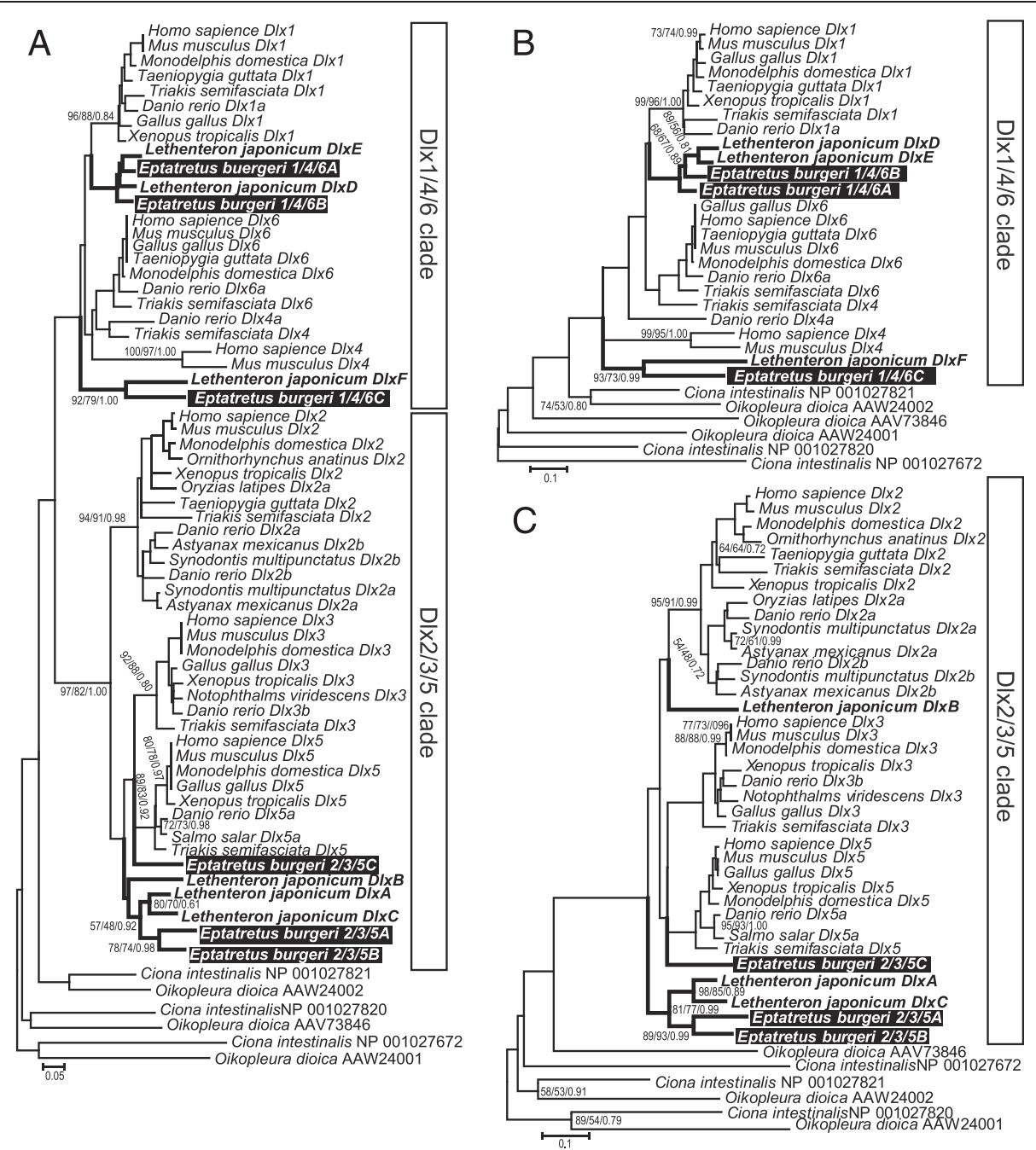

Figure 2 Molecular phylogenetic trees including the hagfish DIx genes. The topologies and branch lengths were calculated with the neighbour-joining method. (A) Phylogenetic tree containing the D/x1/4/6 and D/x2/3/5 genes (based on 77 amino acid sites). (B) Phylogenetic tree of D/x1/4/6 (based on 101 amino acid sites). (C) Phylogenetic tree of D/x2/3/5 (based on 102 amino acid sites). Bootstrap probabilities (NJ and $\mathrm{ML}$ ) greater than 50 and posterior probabilities (BI) greater than 0.5 are shown at each internal branch supported by all three methods, as the left, middle, and right numbers, respectively. The clades of the cyclostome Dlx genes are indicated with bold lines. The hagfish genes are shown in white bold letters in black boxes.

high. This phylogenetic tree indicates that the duplication that produced $E b D l x 1 / 4 / 6 A$ and $E b D l x 1 / 4 / 6 B$ occurred in the hagfish lineage after its separation from the lamprey lineage.

The tree topology of the $D l \times 2 / 3 / 5$ subfamily also suggested an independent gene duplication in the hagfish lineage (Figure 2C; Additional file 2). The cluster containing $E b D l \times 2 / 3 / 5 A$ and $E b D l \times 2 / 3 / 5 B$ formed a sister group with the cluster containing lamprey $D l x A$ and $D l x C$, with high bootstrap support. This cluster was located at the basal position of the $D l \times 2 / 3 / 5$ clade (Figure 2C). In the phylogenetic tree without outgroups, the clade containing $E b D l \times 2 / 3 / 5 A, E b D l \times 2 / 3 / 5 B$, lamprey $D l x A$, and $D l x C$ had a high supporting value in all the phylogenetic methods used (Additional file 2), suggesting that independent gene duplications had occurred in both the lamprey and hagfish lineages. This phylogenetic tree also suggested that the $E b D l \times 2 / 3 / 5 C$ and lamprey $D l x B$ genes are most closely related to each other (Additional file 2).

\section{Embryonic expression patterns of the hagfish DIx genes}

To observe the expression patterns of the hagfish $D l x$ genes in the primordia of the cranial skeleton and inner ear, we used in situ hybridization in the head regions of hagfish embryos at two different stages (designated "middle-" and "late-pharyngula embryos"; Figure 1). In these two embryos, we conducted anatomical observations and identified the exact locations of the otic and optic vesicles and several skeletal tissues, with reference 


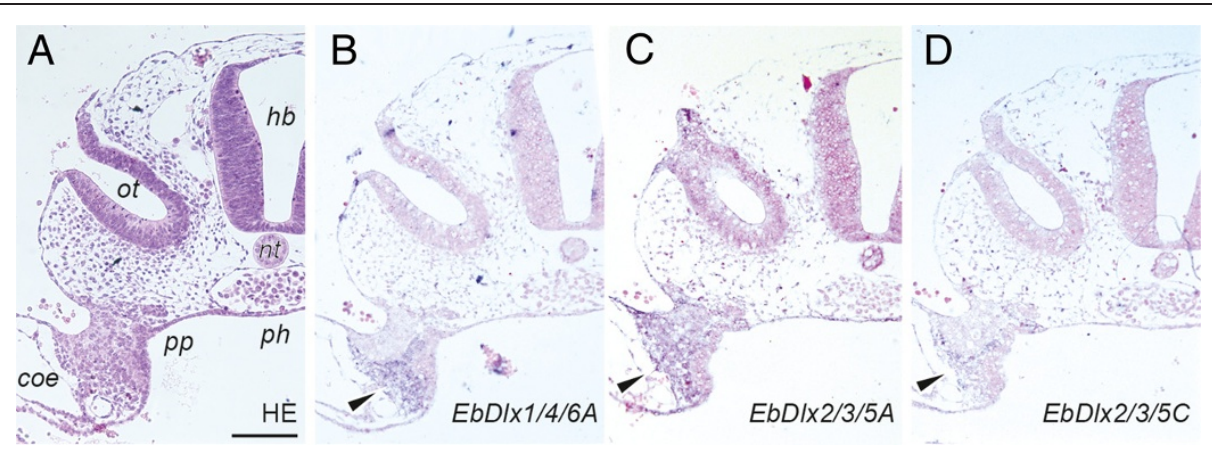

Figure 3 Expression patterns of the Dlx genes in the middle-pharyngula embryo at the otic placode level. (A) Transverse view of a haematoxylin- and eosin-stained section. (B-D) Expression patterns of the D/x genes. DIG-labelled probes for EbD/x1/4/6A (B), EbD/x2/3/5B (C), and EbD/x2/3/5C (D) were detected in the ectomesenchymal cells (arrowheads). The sections used for in situ hybridization were counterstained with ISH Red (B-D). The level of the section is indicated by an arrow in Figure 1A. Abbreviations: coe, coelom; $h b$, hindbrain; $n t$, notochord; ot, otic vesicles; ph, pharynx; pp, pharyngeal pouch. Scale bar, $100 \mu \mathrm{m}$.

to the descriptions and sketches of previous researchers, including Dean (1899) [52], Cole (1905) [20], and Holmgren (1973) [53]. We examined transverse sections at the antero-posterior level containing the otic vesicle, the rostral pharyngeal pouches, and the head mesenchyme in the middle-pharyngula embryo, and at the levels of the otic to pre-otic regions in the latepharyngula embryo, for the expression of all six hagfish $D l x$ genes and the pseudogene (Figures 1, 3, 4 and 5; see also Additional file 3).

In the middle-pharyngula embryo, transcripts of $E b D l x 1 /$ $4 / 6 A, E b D l \times 2 / 3 / 5 A$, and $E b D l \times 2 / 3 / 5 C$ were detected in the mesenchymal cells between the pharyngeal endoderm and the surface ectoderm, which are presumptive cranial neural crest cells (Figure 3). Although not as distinct as the expression patterns of those three genes, $E b D l \times 1 / 4 / 6 B$ and EbDlx2/3/5B tended to be detected in the lateral ventral part of the epithelial cells of the otic vesicles (Additional file 3). In the late-pharyngula embryo at the pre-otic level, which contains the trabecular cartilage (indicated by the arrowhead in Figure 1B; Figure 4A), transcripts of $E b D l \times 1 / 4 / 6 A, E b D l \times 2 / 3 / 5 B$, and $E b D l \times 2 / 3 / 5 C$ were detected (Figure 4B-D). The signal for $E b D l x 1 / 4 / 6 A$ was detected in the mesenchymal cells of the primordium of the tendons of the vagina of the clavatus, which is one of the tissues of the hagfish feeding apparatus (Figure 4B). The expression of $E b D l \times 2 / 3 / 5 B$ was observed in different mesenchymal cells of the primordial tendons and cartilages, including the tendons of the clavatus muscle and the vagina of the clavatus, the basal cartilage, and the cartilage that comprises the trabecula, dorsal longitudinal bar, and extrapalato-quadrate (Figure 4C). A highly intense $E b D l \times 2 / 3 / 5 C$ signal was detected in the mesenchyme located on the ventral aspects of the pharynx and the basal cartilage (Figure 4D). At the level of the inner ear (Figure 1B, arrow), EbDlx1/4/6B and
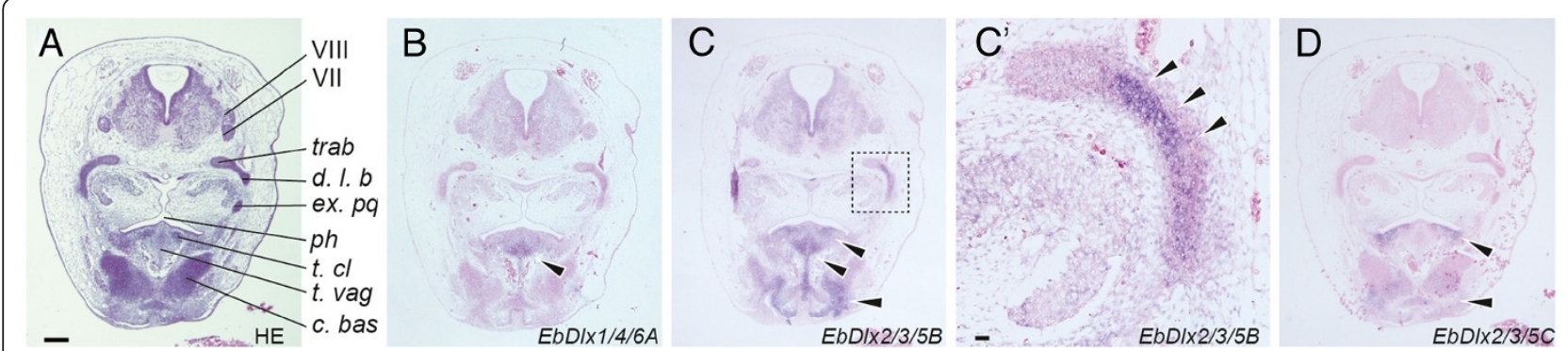

Figure 4 Expression patterns of the Dlx genes in the late-pharyngula embryo at the inner ear level. (A) Transverse view of a haematoxylin- and eosin-stained section. (B-D) Expression patterns of the Dlx genes. The sections for in situ hybridization were counterstained with ISH Red (B-D). (B) Expression of EbD/x1/4/6A was detected in the mesenchymal cells at $t$. vag (arrowhead). (C) EbD/x2/3/5B showed a broad expression pattern in three skeletal elements (t. Cl, t. vag, and c. bas) (arrowheads). (C') Higher magnification of the dotted box shown in C; restricted expression pattern of EbD/x2/3/5B was detected in one cartilaginous complex consisting of $d$. I. b, ex. pq, and trab, (arrowheads). (D) $E b D / \times 2 / 3 / 5 C$ was expressed in the c. bas and mesenchymal cells on the ventral aspect of $p h$. The level of the section is indicated by an arrowhead in Figure 1B. Abbreviations: c. bas, basal cartilage; d. I. b, dorsal longitudinal bar; ex. pq, extrapalato-quadrate; ph, pharynx; t. cl, tendon of the clavatus muscle; $t$. vag, tendon of the vagina of the clavatus; trab, trabecula; VII, facialis nerve; VIII, saccularis nerve. Scale bars, $100 \mu \mathrm{m}$ $(\mathbf{A}) ; 10 \mu \mathrm{m}\left(\mathbf{C}^{\prime}\right)$. 

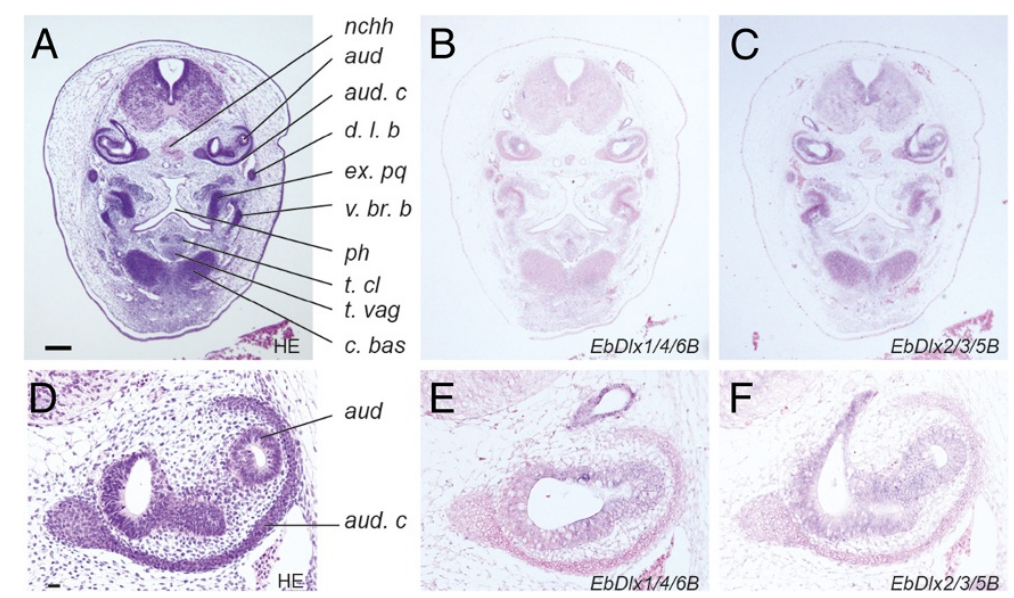

Figure 5 Expression patterns of the Dlx genes in the late-pharyngula embryo at the inner ear level. (A) Transverse view of a haematoxylin- and eosin-stained section. (B, C) Expression patterns of the $D / x$ genes. The sections for in situ hybridization were counterstained with ISH Red (B, C, E, F). (D-F) Higher magnification of the inner ear regions in $\mathbf{A}-\mathbf{C}$, respectively. EbD/x1/4/6B and EbD/x2/3/5B were weakly expressed in aud (E, F). The level of the section is indicated by an arrow in Figure 1B. Abbreviations: aud, auditory capsule; aud. $\boldsymbol{C}$, auditory cartilage; c. bas, basal cartilage; d. I. b, dorsal longitudinal bar; ex. pq, extrapalato-quadrate; nchh, notochordal hook; ph, pharynx; t. $c$, tendon of the clavatus muscle; t. vag, tendon of the vagina of the clavatus; v. br. b, ventral branchial bar. Scale bars, $100 \mu \mathrm{m}$ (A); $10 \mu \mathrm{m}$ (D).

$E b D l x 2 / 3 / 5 B$ showed distinct expression patterns in the auditory capsule (Figure 5). EbDlx2/3/5B was expressed broadly throughout the auditory capsule with homogeneous intensity (Figure 5B, E), whereas EbDlx1/4/6B showed a strong signal on the lateral side of the auditory capsule (Figure 5C, F).

The expression pattern of $E b D l x \Psi$ is broad, and no specific signals were detected in the middle-pharyngula stage. In the late-pharyngula stage, this pseudogene showed specific expression patterns in the cranial cartilages and inner ear (Additional file 3).

\section{Discussion}

In this study, we successfully isolated seven hagfish cDNAs, whose deduced amino acid sequences show significant similarity to the Dlx sequences of the jawed vertebrates. Our molecular phylogenetic analyses suggested that six of these with intact open reading frames are homologues of the $D l x$ genes reported for other vertebrates, and the other is a transcribed pseudogene with a nonsense nucleotide substitution. We also analysed the expression patterns of all six isolated genes in middleand late-pharyngula hagfish embryos. From our results, we deduced the common ancestral state and evolutionary processes of the $D l x$ genes in the cyclostome lineage, based on our previous knowledge of the molecular phylogeny and gene expression patterns of the $D l x$ genes of the lampreys and gnathostomes [7,13,14,38-42].

Although the phylogenetic relationships among all the $D l x$ genes are not fully resolved, the topologies of the phylogenetic trees indicate that some of the hagfish and lamprey $D l x$ genes were duplicated in the cyclostome lineage (Figures 2 and 6). Because $E b D l \times 1 / 4 / 6 A$ and $-B$ and $E b D l x 2 / 3 / 5 A$ and $-B$ form clusters with high bootstrap support, it seems plausible that these genes were duplicated uniquely in the hagfish lineage (Additional file 2). The same assumption can be made for the $D l x A,-C,-D$, and $-E$ genes of the lamprey, as shown previously [51]. Thus, the evolution of these cyclostome $D l x$ genes can be described as follows. First, the $D l \times 1 / 4 / 6 A B, D l \times 2 / 3 / 5 A B$, $D l x 1 / 4 / 6 C$, and $D l x 2 / 3 / 5 C$ genes existed in the genome of the last common ancestor of the hagfish and lampreys (Figure 6; the boxed arrows labelled $D l \times 1 / 4 / 6 A B, D l \times 2 / 3 /$ $5 A B, D l \times 1 / 4 / 6 C$, and $D l \times 2 / 3 / 5 C$ ). Second, at the divergence of the two cyclostome lineages, the hagfish and lampreys, these ancestral cyclostome $D l x$ genes were segregated into the two species lineages (Figure 6; the boxed arrows labelled $D l x 1 / 4 / 6 A B, D l \times 2 / 3 / 5 A B$, and $D l x 2 / 3 / 5 C)$. Third, independent gene duplications in the hagfish and lamprey lineages produced two additional $D l x$ genes in each lineage (Figure 6; the boxed arrows labelled $D l x A, D l x C, D l x D, D l x E, D l x 1 / 4 / 6 A$, $D l x 2 / 3 / 5 A, D l x 1 / 4 / 6 B$, and $D l x 2 / 3 / 5 B)$. To confirm this hypothetical scenario, several problems must be resolved. One is our confidence in the phylogenetic relationships among the cyclostome $D l x$ genes. We cannot completely exclude the possibility that the $D l x$ genes were duplicated at a more ancient point in time, such as in the common ancestor of the cyclostomes. It has previously been suggested that the unique sequence characters of the lamprey genes probably resulted in the exclusive lamprey gene clusters on the phylogenetic trees, erroneously supporting lamprey-lineage-specific gene duplications [52]. This may also have occurred in the hagfish genes. 


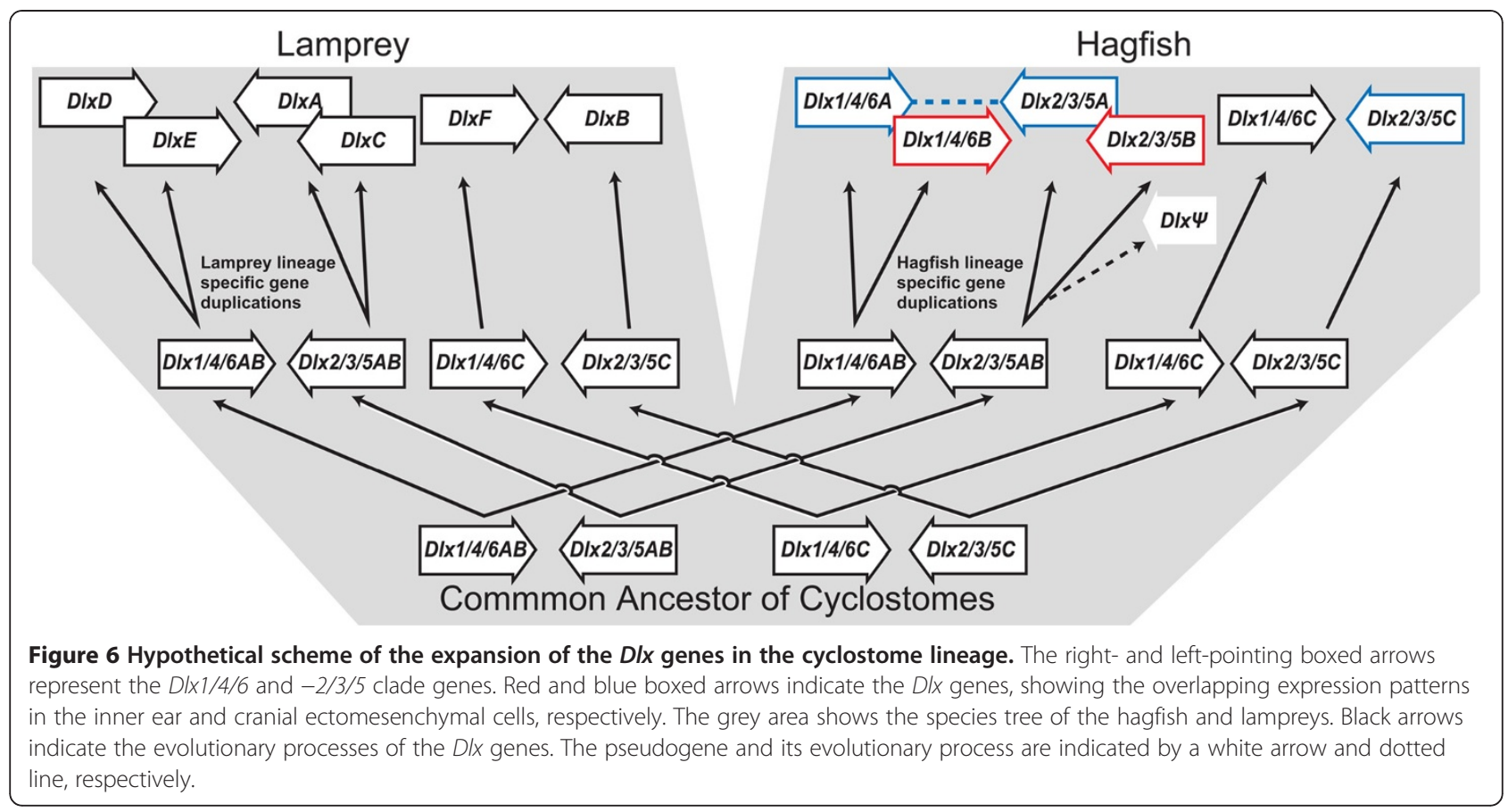

It also remains to be ascertained whether the bi-gene cluster structure of the $D l x$ genes has been conserved in the cyclostomes during the entire course of their evolution. Given that the bi-gene clusters were already present before the divergence of the cyclostomes and gnathostomes $[44,51,54,55]$, it is reasonable to assume that the ancestral cyclostome $D l x$ genes were also present in bi-gene clusters (Figure 6). Our data show that the expression domains of the hagfish $D l x$ genes overlap (Figures 3 and 5). The expression of $E b D l x 1 / 4 /$ $6 A, E b D l \times 2 / 3 / 5 A$, and $E b D l \times 2 / 3 / 5 C$ was detected in the pharyngeal mesenchymal cells of the middle-pharyngula embryo (Figure 3), as is seen in the lampreys and gnathostomes $[7,13,14]$. A number of conserved cis-acting regulatory elements that are shared by two different $D l x$ genes and contribute to their overlapping expression patterns in the pharyngeal mesenchymal cells have been identified in the intergenic regions of the gnathostome $D l x$ gene clusters [56-58]. Therefore, it seems plausible that the hagfish $D l x$ genes that show overlapping expression patterns in the pharyngeal mesenchymal cells have retained the ancestral bi-gene clusters, with conservation of these cis-acting regulatory elements (the blue boxed arrows and the dashed line in Figure 6).

In this context, it is worth considering the evolutionary relationships between the two rounds of wholegenome duplication, the number of $D l x$ genes, and their linkage to other genes. Based on the presumption that the Hox cluster and its associated genes (including the $D l x$ genes), the so-called the "core-Hox paralogon", were increased by the two rounds of genomic duplication in the common ancestor of the vertebrates [46,47], the two $D l x$ genes in each bi-gene cluster are also thought to have been duplicated twice, consequently producing eight $D l x$ genes in total, including the two hypothetical genes ( $D l x 7$ and $D l x 8)$ in the genome of the common vertebrate ancestor. To explain why sharks and mammals have only six $D l x$ genes $(D l x 1-6)$, it is assumed that the two hypothetical $D l x$ genes $(D l x 7$ and $D l x 8)$ that should have been linked to the HoxC cluster and Col2A1 were lost before the radiation of the extant gnathostomes [44]. This raises the question of whether this loss predated the split of the cyclostome lineage from the gnathostome lineage and, if not, whether the genomes of the modern cyclostome species have retained $D l x 7$ and $D l x 8$. To answer this question, further investigation of their whole genomes is required, with particular consideration of whether the $D l x$ genes are localized in the genomic regions containing the $\operatorname{HoxC}$ cluster and Col2A1 genes in the cyclostome genomes. To investigate all these issues, the whole-genome sequence of the hagfish is required.

Recent progress in phylogenetic analysis has allowed us to construct reliable phylogenetic trees, and these trees support the monophyly of the cyclostomes $[25-27,34,59]$. However, identifying the orthology and resolving the molecular phylogeny of many cyclostome genes remain challenging [60]. In fact, our study has shown that the evolutionary processes of the hagfish $D l x$ genes cannot be explained by any simple parsimonious assumption based on a one-to-one orthology between the hagfishes, lampreys, and gnathostomes. Non-parsimonious evolutionary 
processes with "hidden paralogy", involving ancient gene duplications followed by lineage-specific gene losses, have been proposed for several genes, including $C d x$ and Bmp2/4/16 [61]. Therefore, we must be cautious in applying the parsimonious assumption to the evolutionary processes of other cyclostome genes. This caution also seems applicable to the hagfish morphological characters, such as the inner ear and cranial skeleton [19,30], in which the embryonic function of the $D l x$ genes is implicated, although these morphological characters have long been interpreted as plesiomorphic in the classical "Vertebrata", excluding the hagfishes, based on parsimonious assumptions in the field of palaeontology [15-17].

\section{Conclusions}

Our study has shown that the hagfish has retained at least six intact protein-coding $D l x$ genes and a single pseudogene. Conventional molecular phylogenetic methods suggest that four of these were generated by independent gene duplications in the hagfish lineage. These data indicate that more than half the $D l x$ genes of the hagfish are paralogous to the $D l x$ genes of the lampreys and gnathostomes, suggesting a complex gene phylogeny, possibly involving lineage-specific gene losses. The hagfish $D l x$ genes show overlapping embryonic expression patterns, as previously observed in the lampreys and gnathostomes. Our data indicate that the evolutionary processes of the hagfish $D l x$ genes cannot be explained by a simple evolutionary scenario inferred according to the principle of maximum parsimony.

\section{Methods}

Sample collection, aquarium maintenance, and embryonic materials

Adult male and female E. burgeri were collected in the Japan Sea off Shimane and Yamaguchi. The male and female individuals were maintained in an aquarium tank and a cage in the sea according to published methods [29,30,32]. From 2008 to 2009, 42 hagfish embryos were obtained in the aquarium tank and the cage. We gave identification numbers to these embryos [31] and staged them according to Dean's figures and descriptions [52]. Among these embryos, the middle- and late-pharyngula stage embryos (\#0903 and \#B04, respectively) were selected as the most appropriate embryos for histological analysis and the analysis of the expression patterns of the $D l x$ genes.

\section{Molecular cloning}

Molecular cloning and sequencing were performed according to a previous report [30]. A single pharyngula-stage embryo, which was obtained in 2007 [30], was used for total RNA extraction by using TRIzol Reagent (Invitrogen, Japan). Degenerate RT-PCR was performed to amplify the fragment of cDNA encoding the conserved homeobox region of the Dlx amino acid sequence, using three different degenerate primer sets (Additional file 4). The PCR products were isolated on a $2 \%$ agarose gel, and the individual bands were excised from the gel. The amplified PCR fragments containing the conserved homeobox regions of the $D l x$ genes were independently ligated with the TOPO TA Cloning Dual Promoter Kit (Invitrogen) and transformed into Escherichia coli $\mathrm{DH} 5 \alpha$. In total, more than 12 clones were picked from each population of clones and sequenced using an ABI 3130XL automated sequencer (Applied Biosystems, Japan). To determine the full-length cDNA sequences, the $5 /$ and $3 /$ ends were amplified with a GeneRacer kit (Invitrogen) with specific primers (Additional file 4) and sequenced with the method described above. Sequence traces were aligned with CodonCode Aligner (CodonCode Corporation, Dedham, MA, USA). To confirm the stop codon site in EbDlx $\Psi$, PCR and sequencing were performed with three different cDNA samples, which were isolated from different hagfish embryos, using specific primers (Additional file 4). The sequence data were submitted to the DDBJ database [DDBJ:AB679710-AB679716].

\section{Molecular phylogenetic analysis}

A multiple sequence alignment of the $D l x$ genes derived from representative vertebrate species was constructed with the CLUSTALW multiple alignment program [62] (also see Additional file 1) and refined by visual inspection. Based on this alignment, molecular phylogenetic analyses were performed using three different methods: the neighbour-joining (NJ), maximum likelihood (ML), and Bayesian inference (BI) methods. The NJ and ML trees were constructed with 1000 bootstrap replications. JTT and WAG models were used to construct the NJ and ML trees, respectively. The BI analyses were based on two independent runs of two million generations, with samples taken from every 100 generations. Each run consisted of one cold and three heated chains. The NJ, ML, and BI analyses were performed with MEGA [63], PhyML [64], and MrBayes [65], respectively.

\section{Histology and in situ hybridization}

The hagfish embryos and adult specimens were fixed by immersion in Serra's fixative, processed for paraffin sectioning by standard methods, and sectioned to $6-10 \mu \mathrm{m}$. A single section was place on a glass slide, and the adjacent sections were used for haematoxylin and eosin staining and in situ hybridization. The probes were prepared and in situ hybridizations were conducted based on previous reports $[30,66]$. In situ hybridization was performed in a Ventana automated machine (Roche Diagnostics, Japan). To detect the signals, a BlueMap NBT/BCIP substrate kit 
was used, and the samples were counterstained with a nuclear Fast Red equivalent reagent, ISH Red (Roche Diagnostics). We performed several pilot in situ hybridization experiments using the olfactory epithelial tissues of the adult hagfish, because the olfactory epithelium is known to express several $D l x$ genes in mammals $[67,68]$ (Additional file 5). The research followed internationally recognized guidelines. We received ethical approval from RIKEN Kobe Institute Safety Center.

\section{Additional files}

Additional file 1: Multiple alignment of the conserved regions of the $D / x$ genes. Predicted amino acid sequences encoded by the $D / x$ genes of E. burgeri and various other species. Seven hagfish, six lampreys, 49 gnathostomes, and six tunicate Dlx proteins were aligned by CLUSTALW [62] and visual inspections. Identical sites are boxed. The stop codon site of the hagfish D/x pseudogene is indicated by asterisk and arrowhead. Accession numbers are as follows: Astyanax mexicanus DIx2a [ABG89858.1]; Astyanax mexicanus Dlx2b [ABG89859.1]; Ciona intestinalis Dlx homologue [NP_001027821, NP_001027672, NP_001027820]; Danio rerio Dlx1a, NP_571380.1; Danio rerio Dlx2a [CAP19546.1]; Danio rerio Dlx2b [NP_571372.1]; Danio rerio Dlx3b [NP_571397.2]; Danio rerio Dlx4a [NP_571375.1]; Danio rerio Dlx5a [AAH83280.1]; Danio rerio Dlx6a [NP_571398.1]; Eptatretus burgeri Dlx1/4/6A [AB679710]; Eptatretus burgeri Dlx1/4/6B [AB679711]; Eptatretus burgeri Dlx1/4/6C [AB679712]; Eptatretus burgeri DIx2/3/5A [AB679713]; Eptatretus burgeri Dl×2/3/5B [AB679714]; Eptatretus burgeri Dlx2/3/5C [AB679715]; Eptatretus burgeri Dlx pseudo [AB679716]; Gallus gallus Dlx1 [NP_001039307.2]; Gallus gallus Dl×3 [NP_990135.1]; Gallus gallus Dlx5 [NP_989490.1]; Gallus gallus Dlx5 [NP_989490.1]; Gallus gallus Dlx6 [NP_001074359.1]; Homo sapiens Dlx [NP_835221.2]; Homo sapiens DIx2 [NP_004396.1]; Homo sapiens Dlx3 [NP_005211.1]; Homo sapiens Dlx4 [NP_612138.1]; Homo sapiens Dlx5 [NP_005212.1]; Homo sapiens DIx6 [NP_005213.2]; Lethenteron japonicum DIxA [AB292628]; Lethenteron japonicum DIxB [AB292629]; Lethenteron japonicum DlxC [AB292630]; Lethenteron japonicum DIxD [AB048759]; Lethenteron japonicum DIxE [AB048759]; Lethenteron japonicum DIxF [AB292633]; Monodelphis domestica DIX1 [XP_001368011.1]; Monodelphis domestica Dlx2 [XP_001368046.1]; Monodelphis domestica Dl×3 [XP_001367695.1]; Monodelphis domestica Dlx5 [XP_001363081.1]; Monodelphis domestica Dlx6 [XP_001363167.1]; Mus musculus Dlx1 [NP_034183.1]; Mus musculus Dlx2 [NP_034184]; Mus musculus Dlx3 [NP_034185.1]; Mus musculus Dlx4 [NP_031893.3]; Mus musculus DIx5 [NP_034186.2]; Mus musculus Dlx6 [NP_034187]; Notophthalmus viridescens Dl×3 [P53770.1]; Oikopleura dioica Dlx homologues, [AAW24001, AAW24002, AAV73846]; Ornithorhynchus anatinus Dlx2 [XP_001514642.1]; Oryzias latipes Dlx2a [NP_001098290.1]; Petromyzon marinus DIxA [AAG41495.1]; Petromyzon marinus DIxB [AAG41496.1]; Petromyzon marinus DlxC [AAG41497.1]; Petromyzon marinus DIxD [AAG41498.1]; Salmo salar Dlx5a [NP_001134142.1]; Salmo salar Dlx5a [NP_001134142.1]; Synodontis multipunctatus DIx2a [ABG89865.1]; Synodontis multipunctatus Dlx2b [ABG89866.1]; Taeniopygia guttata DIx1 [XP_002198787.1]; Taeniopygia guttata Dlx2 [XP_002196070.1]; Taeniopygia guttata Dlx6 [XP_002197360.1]; Triakis semifaciata Dlx1 [AAV85983.1]; Triakis semifaciata Dlx2 [AAV85984.1]; Triakis semifaciata Dlx3 [AAV85985.1]; Triakis semifaciata DIx4 [AAV85986.1]; Triakis semifaciata Dlx5 [AAV85987.1]; Triakis semifaciata Dlx6 [AAV85988.1]; Xenopus tropicalis DIx1 [NP_001093727.1]; Xenopus tropicalis DIx2 [NP_001008061.1]; Xenopus tropicalis DIx3 [NP_001025566.1]; Xenopus tropicalis DIx5 [NP_001004778.1]; Xenopus tropicalis DIx5 [NP_001004778.1].

Additional file 2: Unrooted molecular phylogenetic trees including the hagfish Dlx genes. The topologies and branch lengths were calculated with the neighbour-joining method. (A) Phylogenetic tree of D/x1/4/6 (based on 155 amino acid sites). (B) Phylogenetic tree of D/x2/3/ 5 (based on 106 amino acid sites). Bootstrap probabilities (NJ and ML) greater than 50 and posterior probabilities (BI) greater than 0.5 are shown at each internal branch supported by all three phylogenetic trees, as the left, middle, and right numbers, respectively. The clades of the cyclostome D/x genes are indicated with bold lines. The hagfish genes are shown in white letters in a black box.

Additional file 3: The expression patterns of the $D / x$ genes in middle- and late-pharyngula embryos of $E$. burgeri. The sections of the middle-pharyngula embryos $\left(\mathrm{A}-\mathrm{H}^{\prime}\right)$ and the late-pharyngula embryos $\left(I-X^{\prime}\right)$ were derived from the embryos in Figure 1. Higher magnification of cranial cartilaginous tissues (trab and d.l.b) and inner ear in the latepharyngeal embryos are shown in the third $\left(J^{\prime}-P^{\prime}\right)$ and fifth rows $\left(Q^{\prime}-X^{\prime}\right)$, respectively. Haematoxylin- and eosin-stained sections ( $\mathrm{A}, \mathrm{l}, \mathrm{I}^{\prime}, \mathrm{Q}$, and $\mathrm{Q}^{\prime}$ ) were obtained from the same level as the sections used for in situ hybridization with DIG-labelled probes for EbD/x1/4/6A (B, J, J', R, and R'), $E b D / x 1 / 4 / 6 B\left(C, K, K^{\prime}, S\right.$, and $\left.S^{\prime}\right), E b D / x 1 / 4 / 6 C\left(D, L, L^{\prime}, T\right.$, and $\left.T^{\prime}\right), E b D / x 2 / 3 / 5 A$ $\left(E, M, M^{\prime}, U\right.$, and $\left.U^{\prime}\right), E b D / \times 2 / 3 / 5 B\left(F, N, N^{\prime}, V\right.$, and $\left.V^{\prime}\right), E b D / \times 2 / 3 / 5 C\left(G, O, O^{\prime}\right.$, W, and $\left.W^{\prime}\right)$, and $E b D I x \Psi\left(H, P, P^{\prime}, X\right.$, and $\left.X^{\prime}\right)$. The sections for in situ hybridization were counterstained with ISH Red. The signals from the probes in the mesenchymal cells $(B, C, G$, and $O$ ) and the cartilaginous primordium $\left(\mathrm{N}^{\prime}\right)$ are indicated by arrowheads. Abbreviations: aud, auditory capsule; aud. c, auditory cartilage; c. bas, basal cartilage; coe, coelom; hb, hindbrain; d. I. b, dorsal longitudinal bar; ex. pq, extrapalatoquadrate; $n c h h$, notochordal hook; $n t$, notochord; ot, otic vesicles; $p h$, pharynx; pp, pharyngeal pouch; $t . c l$, tendon of the clavatus muscle; $t$. vag, tendon of the vagina of the clavatus; trab, trabecular; $v$. br. $b$, ventral branchial bar; VII, facialis nerve; VIII, saccularis nerve. Scale bars, 100 mm.

\section{Additional file 4: List of primer sets.}

Additional file 5: Comparison of the expression patterns of the $D / x$ genes in the adult olfactory epithelium of $E$. burgeri. Haematoxylinand eosin-stained sections (A) were obtained from the same level as the sections used for in situ hybridization with DIG-labelled probes for $E b D / x 1 / 4 / 6 A(B), E b D / \times 1 / 4 / 6 B(C), E b D / x 1 / 4 / 6 C(D), E b D / \times 2 / 3 / 5 A(E), E b D / \times 2 /$ 3/5B (F), EbD/x2/3/5C (G), and EbDlx $\psi(H)$. The sections for in situ hybridization were counterstained with ISH Red. All the genes were expressed in the olfactory epithelium. EbD/x1/4/6A, EbD/x2/3/5B, and $E b D / \times 2 / 3 / 5 C$ were detected in a few cells across the entire region of the olfactory epithelium. Strongly EbD/x1/4/6C-positive cells tended to be located at the levels of the non-basal olfactory epithelium cells. EbD/x2/3/ $5 A$ showed strong expression in the basal cells. Subtle expression of $E b D / \times 1 / 4 / 6 B$ was detected in the entire region of the olfactory epithelium. EbDlx $\psi$ showed a broad expression pattern in the basal cells and olfactory epithelium. The layers in which the probe signals were detected are indicated by brackets on the left side of the panels $(B-H)$.

Abbreviations: bc, basal cells; oe, olfactory epithelium. Scale bar, $100 \mu \mathrm{m}$.

\section{Competing interests}

The authors declare that they have no competing interests.

\section{Authors' contributions}

SF conducted intensive molecular cloning of the D/x genes and in situ hybridization. YO and KGO collected the samples and maintained the aquarium tank. KGO and S. Kuraku analysed the molecular sequence data. KGO wrote the first draft of the manuscript. KGO, S. Kuraku, and S. Kuratani contributed to the writing of the final version of the manuscript. All authors discussed the results and commented on the manuscript. All authors read and approved the final manuscript.

\section{Authors' information}

$\mathrm{SF}$ is a technical assistant and $\mathrm{YO}$ is a doctoral student in the Laboratory for Evolutionary Morphology at the Center for Developmental Biology (CDB). KGO initiated this study in the same laboratory but now holds an independent position at the Yilan Marine Research Station, ICOB, Academia Sinica in Taiwan. S. Kuraku is the leader of the Genome Resource and Analysis Unit at CDB. S. Kuratani is the director of the Laboratory for Evolutionary Morphology at CDB.

\section{Acknowledgements}

We are grateful to Captain Osamu Kakitani and the members of the Fishery Association in Gotsu City and to Kiyomi Kayano (Director, Sekikatsu Inc.) for 
their assistance in collecting the hagfish. We also thank Fumiaki Sugahara at the Laboratory for Evolutionary Morphology, CDB, RIKEN, for maintaining the aquariums, and the staff of Yilan Marine Station, ICOB, Academia Sinica, for their assistance with administrative support. Finally, we wish to express our deep gratitude to Kenta Sumiyama and Kyle Martin for their advice on the experimental strategy. This research was supported by Grants-in-Aid for Scientific Research from the Japan Society for the Promotion of Science (JSPS), Ministry of Education, Culture, Sports, Science, and Technology of Japan, and NSC grant 101-2311-B-001-001-MY2 from the National Science Council of Taiwan.

\section{Author details}

'Laboratory for Evolutionary Morphology, RIKEN Center for Developmental Biology, Kobe 650-0047, Japan. ²Department of Biology, Graduate School of Science, Kobe University, Kobe, Japan. ${ }^{3}$ Genome Resource and Analysis Unit, RIKEN Center for Developmental Biology, Kobe 650-0047, Japan. ${ }^{4}$ Laboratory of Aquatic Zoology, Marine Research Station, Institute of Cellular and Organismic Biology, Academia Sinica, Yilan 26242, Taiwan.

Received: 5 September 2012 Accepted: 11 January 2013

Published: 19 January 2013

\section{References}

1. Gans C, Northcutt RG: Neural crest and the origin of vertebrates: a new head. Science 1983, 220:268-274.

2. Northcutt GR: The new head hypothesis revisited. J Exp Zool B Mol Dev Evol 2005, 304B:274-297.

3. Kardong K: Vertebrates Comparative Anatomy, Function, Evolution. 5th edition. New York: McGraw Hill Higher Education; 2008.

4. Ogasawara M, Shigetani Y, Suzuki S, Kuratani S, Satoh N: Expression of thyroid transcription factor-1 (TTF-1) gene in the ventral forebrain and endostyle of the agnathan vertebrate, Lampetra japonica. Genesis 2001, 30:51-58.

5. Murakami Y, Ogasawara M, Sugahara F, Hirano S, Satoh N, Kuratani S: Identification and expression of the lamprey Pax6 gene: evolutionary origin of the segmented brain of vertebrates. Development 2001, 128:3521-3531.

6. Myojin M, Ueki T, Sugahara F, Murakami Y, Shigetani Y, Aizawa S, Hirano S, Kuratani S: Isolation of Dlx and Emx gene cognates in an agnathan species, Lampetra japonica, and their expression patterns during embryonic and larval development: conserved and diversified regulatory patterns of homeobox genes in vertebrate head evolution. J Exp Zool 2001, 291:68-84

7. Neidert AH, Virupannavar V, Hooker GW, Langeland JA: Lamprey D/x genes and early vertebrate evolution. Proc Natl Acad Sci U S A 2001, 98:1665-1670.

8. Uchida K, Murakami Y, Kuraku S, Hirano S, Kuratani S: Development of the adenohypophysis in the lamprey: evolution of epigenetic patterning programs in organogenesis. J Exp Zoolog B Mol Dev Evol 2003, 300:32-47.

9. McCauley DW, Bronner-Fraser M: Conservation and divergence of BMP2/4 genes in the lamprey: expression and phylogenetic analysis suggest a single ancestral vertebrate gene. Evol Dev 2004, 6:411-422.

10. Takio Y, Pasqualetti M, Kuraku S, Hirano S, Rijli FM, Kuratani S: Evolutionary biology: lamprey Hox genes and the evolution of jaws. Nature 2004, 429: 1 p following 262

11. McCauley DW, Bronner-Fraser M: Importance of SoxE in neural crest development and the evolution of the pharynx. Nature 2006, 441:750-752.

12. Takio Y, Kuraku S, Murakami Y, Pasqualetti M, Rijli FM, Narita Y, Kuratani S, Kusakabe R: Hox gene expression patterns in Lethenteron japonicum embryos-insights into the evolution of the vertebrate Hox code. Dev Biol 2007, 308:606-620.

13. Kuraku S, Takio Y, Sugahara F, Takechi M, Kuratani S: Evolution of oropharyngeal patterning mechanisms involving $D / x$ and endothelins in vertebrates. Dev Biol 2010, 341:315-323.

14. Cerny R, Cattell M, Sauka-Spengler T, Bronner-Fraser M, Yu F, Medeiros DM: Evidence for the prepattern/cooption model of vertebrate jaw evolution. Proc Natl Acad Sci U S A 2011, 107:17262-17267.

15. Forey $P$, Janvier $P:$ Agnathans and the origin of jawed vertebrates. Nature 1993, 361:129-134.
16. Shu D, Morris SC, Zhang ZF, Liu JN, Han J, Chen L, Zhang XL, Yasui K, Li Y: A new species of yunnanozoan with implications for deuterostome evolution. Science 2003, 299:1380-1384.

17. Gess RW, Coates Ml, Rubidge BS: A lamprey from the Devonian period of South Africa. Nature 2006, 443:981-984.

18. Janvier P: Early vertebrates and their extant relatives. In Early vertebrates. Edited by Janvier P. New York: Oxford University Press; 1996:83-225.

19. Janvier P: Evolutionary biology: born-again hagfishes. Nature 2007, 446:622-623.

20. Cole FJ: A monograph on the general morphology of myxinoid fishes, based on a study of myxine. Part 1 . The anatomy of the skeleton. Trans $R$ Soc Edin 1905, XLI(Part III (No. 30)):749-791.

21. Conel JL: The origin of the neural crest. J Comp Neurol 1942, 76:191-215.

22. Jørgensen J, Lomholt JP, Weber RE, Malte $\mathrm{H}$ : The biology of hagfishes. Cambrige: Chapman and Hall Ltd; 1998.

23. Stock DW, Whitt GS: Evidence from 185 ribosomal RNA sequences that lampreys and hagfishes form a natural group. Science 1992, 257:787-789.

24. Mallatt J, Sullivan J: $28 \mathrm{~S}$ and $18 \mathrm{~S}$ rDNA sequences support the monophyly of lampreys and hagfishes. Mol Biol Evol 1998, 15:1706-1718

25. Kuraku S, Hoshiyama D, Katoh K, Suga H, Miyata T: Monophyly of lampreys and hagfishes supported by nuclear DNA-coded genes. J Mol Evol 1999, 49:729-735

26. Furlong RF, Holland PW: Bayesian phylogenetic analysis supports monophyly of ambulacraria and of cyclostomes. Zoolog Sci 2002, 19:593-599.

27. Takezaki N, Figueroa F, Zaleska-Rutczynska Z, Klein J: Molecular phylogeny of early vertebrates: monophyly of the agnathans as revealed by sequences of 35 genes. Mol Biol Evol 2003, 20:287-292.

28. Nicholls H: Evolution: mouth to mouth. Nature 2009, 461:164-166.

29. Ota KG, Kuratani S: The history of scientific endeavors towards understanding hagfish embryology. Zoolog Sci 2006, 23:403-418,

30. Ota KG, Kuraku S, Kuratani S: Hagfish embryology with reference to the evolution of the neural crest. Nature 2007, 446:672-675.

31. Ota KG, Fujimoto S, Oisi Y, Kuratani S: Identification of vertebra-like elements and their possible differentiation from sclerotomes in the hagfish. Nat Commun 2011, 2:373.

32. Ota KG, Kuratani S: Developmental biology of hagfishes, with a report on newly obtained embryos of the Japanese inshore hagfish, Eptatretus burgeri. Zoolog Sci 2008, 25:999-1011.

33. Janvier P: Comparative anatomy: All vertebrates do have vertebrae. Curr Biol 2011, 21:R661-R663.

34. Heimberg AM, Cowper-Sallari R, Semon M, Donoghue PCJ, Peterson KJ: microRNAs reveal the interrelationships of hagfish, lampreys, and gnathostomes and the nature of the ancestral vertebrate. Proc Natl Acad Sci U S A 2010, 107:19379-19383.

35. Gai Z, Donoghue PCJ, Zhu M, Janvier P, Stampanoni M: Fossil jawless fish from China foreshadows early jawed vertebrate anatomy. Nature 2011, 476:324-327.

36. Kuraku S, Kuratani S: Time scale for cyclostome evolution inferred with a phylogenetic diagnosis of hagfish and lamprey cDNA sequences. Zoolog Sci 2006, 23:1053-1064

37. Furlong RF, Younger R, Kasahara M, Reinhardt R, Thorndyke M, Holland PW: A degenerate ParaHox gene cluster in a degenerate vertebrate. Mol Biol Evol 2007, 24:2681-2686.

38. Panganiban G, Rubenstein JL: Developmental functions of the Distal-less/ D/x homeobox genes. Development 2002, 129:4371-4386.

39. Depew MJ, Lufkin T, Rubenstein JL: Specification of jaw subdivisions by Dlx genes. Science 2002, 298:381-385.

40. Solomon KS, Fritz A: Concerted action of two dlx paralogs in sensory placode formation. Development 2002, 129:3127-3136.

41. Depew MJ, Simpson CA, Morasso M, Rubenstein JL: Reassessing the DlX code: the genetic regulation of branchial arch skeletal pattern and development. J Anat 2005, 207:501-561.

42. Brown ST, Wang J, Groves AK: Dlx gene expression during chick inner ear development. J Comp Neurol 2005, 483:48-65.

43. McGuinness T, Porteus MH, Smiga S, Bulfone A, Kingsley C, Qiu M, Liu JK, Long JE, Xu D, Rubenstein JL: Sequence, organization, and transcription of the $D / x-1$ and D/x-2 locus. Genomics 1996, 35:473-485.

44. Stock DW: The Dlx gene complement of the leopard shark, Triakis semifasciata, resembles that of mammals: implications for genomic and morphological evolution of jawed vertebrates. Genetics 2005, 169:807-817. 
45. Morvan-Dubois G, Le Guellec D, Garrone R, Zylberberg L, Bonnaud L: Phylogenetic analysis of vertebrate fibrillar collagen locates the position of zebrafish alpha3(I) and suggests an evolutionary link between collagen alpha chains and hox clusters. J Mol Evol 2003, 57:501-514.

46. Zhang G, Cohn MJ: Genome duplication and the origin of the vertebrate skeleton. Curr Opin Genet Dev 2008, 18:387-393.

47. Lynch VJ, Wagner GP: Multiple chromosomal rearrangements structured the ancestral vertebrate Hox-bearing protochromosomes. PLOS Genet 2009, 5:e1000349.

48. Debiais-Thibaud M, Oulion S, Bourrat F, Laurenti P, Casane D, Borday-Birraux $V$ : The homology of odontodes in gnathostomes: insights from Dlx gene expression in the dogfish, Scyliorhinus canicula. BMC Evol Biol 2011, 11:307.

49. Akimenko MA, Ekker M, Wegner J, Lin W, Westerfield M: Combinatorial expression of three zebrafish genes related to distal-less: part of a homeobox gene code for the head. J Neurosci 1994, 14:3475-3486.

50. Renz AJ, Gunter HM, Fischer JM, Qiu H, Meyer A, Kuraku S: Ancestral and derived attributes of the dlx gene repertoire, cluster structure and expression patterns in an African cichlid fish. EvoDevo 2011, 2:1.

51. Kuraku S, Meyer A, Kuratani S: Timing of genome duplications relative to the origin of the vertebrates: did cyclostomes diverge before or after? Mol Biol Evol 2009, 26(1):47-59.

52. Dean B: On the embryology of Bdellostoma stouti. A genera account of myxinoid development from the egg and segmentation to hatching. Festschrift zum 70ten Geburststag Carl von Kupffer 1899, :220-276.

53. Holmgren N: On two embryos of Myxine glutinosa. Acta Zoologica 1946, 27:1-90.

54. Qiu H, Hildebrand F, Kuraku S, Meyer A: Unresolved orthology and peculiar coding sequence properties of lamprey genes: the KCNA gene family as test case. BMC Genomics 2011, 12:325.

55. Quint E, Zerucha T, Ekker M: Differential expression of orthologous D/x genes in zebrafish and mice: implications for the evolution of the D/x homeobox gene family. J Exp Zool 2000, 288:235-241.

56. Irvine SQ, Cangiano MC, Millette BJ, Gutter ES: Non-overlapping expression patterns of the clustered DII-A/B genes in the ascidian Ciona intestinalis. J Exp Zool B Mol Dev Evol 2007, 308:428-441.

57. Sumiyama K, Irvine SQ, Stock DW, Weiss KM, Kawasaki K, Shimizu N, Shashikant CS, Miller W, Ruddle FH: Genomic structure and functional control of the D/x3-7 bigene cluster. Proc Natl Acad Sci U S A 2002, 99:780-785.

58. Ghanem N, Jarinova O, Amores A, Long Q, Hatch G, Park BK, Rubenstein JL, Ekker M: Regulatory roles of conserved intergenic domains in vertebrate Dlx bigene clusters. Genome Res 2003, 13:533-543.

59. Zerucha T, Stuhmer T, Hatch G, Park BK, Long Q, Yu G, Gambarotta A, Schultz JR, Rubenstein JL, Ekker M: A highly conserved enhancer in the D/x5/D/x6 intergenic region is the site of cross-regulatory interactions between Dlx genes in the embryonic forebrain. J Neurosci 2000 20:709-721

60. Kuraku S: Insights into cyclostome phylogenomics: pre-2R or post-2R. Zoolog Sci 2008, 25:960-968.

61. Kuraku S: Palaeophylogenomics of the vertebrate ancestor-impact of hidden paralogy on hagfish and lamprey gene phylogeny. Integr Comp Biol 2010, 50(1):124-129.

62. Thompson JD, Higgins DG, Gibson TJ: CLUSTAL W: improving the sensitivity of progressive multiple sequence alignment through sequence weighting, position-specific gap penalties and weight matrix choice. Nucleic Acids Res 1994, 22:4673-4680.

63. Kumar S, Nei M, Dudley J, Tamura K: MEGA: a biologist-centric software for evolutionary analysis of DNA and protein sequences. Brief Bioinform 2008, 9:299-306.

64. Guindon S, Gascuel O: A simple, fast, and accurate algorithm to estimate large phylogenies by maximum likelihood. Syst Biol 2003, 52:696-704.

65. Ronquist F, Huelsenbeck JP: MrBayes 3: bayesian phylogenetic inference under mixed models. Bioinformatics 2003, 19:1572-1574.

66. Ota KG, Kuratani S: Expression pattern of two collagen type 2 alpha1 genes in the Japanese inshore hagfish (Eptatretus burgeri) with special reference to the evolution of cartilaginous tissue. J Exp Zool B Mol Dev Evol 2010, 314:157-165.
67. Saino-Saito $S$, Berlin R, Baker $H$ : Dlx-1 and Dlx-2 expression in the adult mouse brain: relationship to dopaminergic phenotypic regulation. J Comp Neurol 2003, 461:18-30.

68. Long JE, Garel S, Alvarez-Dolado M, Yoshikawa K, Osumi N, Alvarez-Buylla A, Rubenstein JL: Dlx-dependent and -independent regulation of olfactory bulb interneuron differentiation. J Neurosci 2007, 27:3230-3243.

doi:10.1186/1471-2148-13-15

Cite this article as: Fujimoto et al:: Non-parsimonious evolution of hagfish Dlx genes. BMC Evolutionary Biology 2013 13:15.

\section{Submit your next manuscript to BioMed Central and take full advantage of:}

- Convenient online submission

- Thorough peer review

- No space constraints or color figure charges

- Immediate publication on acceptance

- Inclusion in PubMed, CAS, Scopus and Google Scholar

- Research which is freely available for redistribution

Submit your manuscript at www.biomedcentral.com/submit
C Biomed Central 